\title{
Acute Mesenteric Lymphadenitis, A Differential Diagnosis of Acute Appendicitis in Adults Also: Case Report
}

\author{
Khalid Mazine $^{1^{*}}$, Ihssane Cherrabi $^{2}$, Khalid Ait Taleb ${ }^{1}$
}

${ }^{1}$ Department of visceral surgery Hassan II university hospital Fez, Morocco

${ }^{2}$ Anesthesiology and Reanimation Department Hassan II university hospital Fez, Morocco

DOI: $10.36347 /$ sasjs.2020.v06i07.006

| Received: 08.07.2020 | Accepted: 16.07.2020 | Published: 28.07.2020

*Corresponding author: MAZINE Khalid

Abstract

Mesenteric lymphadenitis is a self-limiting inflammatory condition affecting the mesenteric lymph nodes, whose presentation mimics appendicitis or intussusception. It typically occurs in children and adolescents. White blood count and C-reactive protein are of limited usefulness in distinguishing between patients with and without mesenteric lymphadenitis. Ultrasonography and or CT scan, the mainstay of diagnosis, discloses 3 or more mesenteric lymph nodes with a short-axis diameter of $8 \mathrm{~mm}$ or more without any identifiable underlying inflammatory process. Once the diagnosis is established, supportive care including hydration and pain medication is advised. Furthermore, it is crucial to reassure patients and families by explaining the condition and stating that affected patients recover completely without residuals within 2-4 weeks. We report the case of a 21 years old female admitted to the surgical emergency for abdominal pain in the right iliac fossa suspecting an appendicitis which found to be an acute mesenteric lymphadenitis.

Keywords: Mesenteric lymphadenitis, acute appendicitis.

Copyright @ 2020: This is an open-access article distributed under the terms of the Creative Commons Attribution license which permits unrestricted use, distribution, and reproduction in any medium for non-commercial use (NonCommercial, or CC-BY-NC) provided the original author and source are credited.

\section{INTRODUCTION}

Mesenteric Lymphadenitis is described as the set of three or more lymph nodes measuring more than $5 \mathrm{~mm}$ in the mesentery of the lower right quadrant of the abdomen, visualized on CT or ultrasonography. It usually occurs in children and adolescents under 16 years [1]. It can be divided into two distinct groups: primary (idiopathic) and secondary due to infectious, malignant or inflammatory disorders.

Recently performed imaging studies among subjects with suspected appendicitis or intussusception confirm that acute mesenteric lymphadenitis is the most frequent alternative diagnosis in children [2], this situation remains less common among adults. Although liberal use of high-quality imaging studies now better characterizes mesenteric lymphadenitis, its natural history and appropriate management have not been clearly defined.

The aim of this case report is to highlight the characteristics of this unusual condition in adult, as well as the differential diagnoses and treatment approaches available.

\section{CASE REPORT}

We report the case of a 21 years old young female originally from Finland traveling to Morocco for tourism; she was referred to our observation for periumbilical abdominal pain for 2 days, with abrupt onset and moderate intensity, which gave away with the use of a common analgesic. It evolved to the right iliac fossa and increased in intensity. She also reported $38^{\circ} \mathrm{C}$ fever, anorexia and vomiting. At physical examination, the abdomen was typical, with superficial and deep palpation pain in right iliac fossa and negative Blumberg's signal. According to the clinical findings, the first diagnosis suggested was an appendicitis. We admitted the patient to the surgical department for diagnostic clarification. We first started by making a blood test which revealed 18000 leucocytes with $75 \%$ of neutrophils, C reactive protein was 220. Ultrasonography discloses at least 5, enlarged (more than $5 \mathrm{~mm}$ short-axis diameter), hypoechoic mesenteric lymph nodes, with no thickened blind-ending tubular structure in the right lower quadrant. The CT of abdomen and pelvis with intravenous contrast (Figure 1) showed a normal appendix, many mesenteric lymphadenopathies in the right-side without any identifiable acute inflammatory process. 
Considering the imaging findings, the hypothesis of mesenteric lymphadenitis was considered. We started an antibiotic treatment with ciprofloxacin, the patient reported an improvement in pain and symptoms after 24 hours of the first dose. She was discharged after 72 hours of hospitalization.
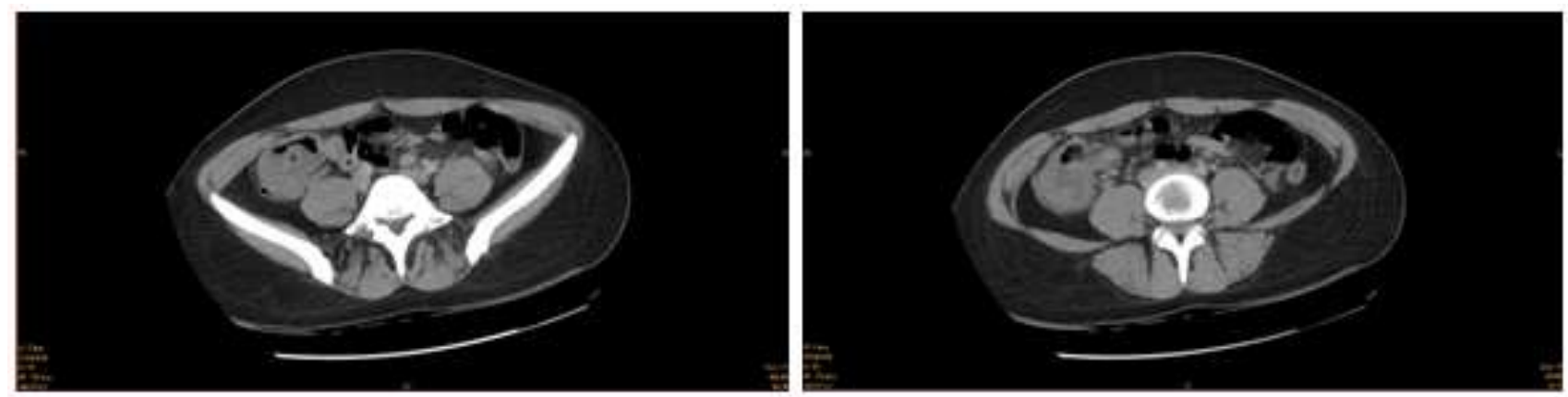

Fig-1: Abdominal CT

\section{DISCUSSION}

For a long time, enlarged mesenteric lymph nodes in the young were considered invariably due to tuberculosis. A few years after the First World War, the existence of a mesenteric lymphadenitis, as an independent clinical entity of nontuberculous origin, was recognized [3]. It was first described by Wilensky et al. [4] and was soon followed by several publications of case series. It occurs most commonly between the ages of 3 and 15 years, with a peak incidence at both extremities of this age group, whilst mesenteric lymphadenitis is distinctly uncommon after the age of 20 years [5].

Acute mesenteric lymphadenitis is an inflammatory process characterized by a cluster of three or more lymph nodes with short-axis diameter of $5 \mathrm{~mm}$ or more in the right lower quadrant and in the paraaortic region without an identifiable acute inflammatory process [6]. Reactive mesenteric lymph node enlargement can occur in conjunction with localized or systemic inflammatory processes, such as acute appendicitis, diverticulitis, cholecystitis, inflammatory bowel disease. Generally, the nodes are not as numerous nor as large as those visualized in patients with mesenteric lymphadenitis [7].

Acute mesenteric lymphadenitis also can occur as a site of infection of several enteric and systemic pathogens, including Yersinia enterocolitica, Yersinia pseudotuberculosis, Bartonella henselae, Salmonella spp, Nocardia spp, Ascaris lumbricoides, Cryptococcus spp, Mycobacterium spp, and HIV [6, 1].

Relevant symptoms and signs of mesenteric lymphadenitis include [7]

- Fever ranges between 38.0 and $38.5^{\circ} \mathrm{C}$, vomiting, and shifts in stool frequency and consistency are frequently reported.
- Pain is usually severe, but, as a rule, the patient does not appear to be severely prostrated. The distribution of pain, like that of appendicitis, is felt both in the periumbilical region and in the right iliac fossa, tenderness is maximal in the right iliac fossa but is often present higher up towards the epigastrium, true abdominal rigidity is usually absent.

All these similarities make the clinical differentiation between mesenteric lymphadenitis and acute appendicitis very challenging. It is necessary to use complementary diagnostic methods to investigate the cause of the signs and symptoms presented. Abdominal ultrasonography is the mainstay of diagnosis, the radiological definition for mesenteric lymphadenitis is a cluster of three or more lymph nodes with short-axis diameter of $5 \mathrm{~mm}$ or more in the right lower quadrant without an identifiable acute inflammatory process [8].

In the case reported the signs were similar to those observed in appendicitis, the US showed the adenopathy, also found in some cases of appendicitis, we performed a CT which confirmed the diagnose of mesenteric lymphadenitis.

The treatment, include hydration and pain medication. Even more crucial is explaining the diagnosis in a clear and logical way (the presence of enlarged lymph nodes is often a source of anxiety because of the association with malignancy) [7]. Mesenteric lymphadenitis is reported to be a selflimited disease and antibiotics are not needed, unless patients are severely ill and show signs of septicemia [9].

In our case, antibiotic therapy was chosen because, given the clinical picture and the results evidenced in the complementary tests, bacterial etiology was the most likely hypothesis [10]. 


\section{CONCLUSION}

Mesenteric lymphadenitis is considered the most common cause of pseudo-appendicular syndrome in children but remains rare in adult.

Differentiation between acute appendicitis and mesenteric lymphadenitis remains challenging even for the astute physicians.

Our case highlight that, despite its rarity, mesenteric lymphadenitis is not an unusual diagnosis in adult, and must not be neglected.

\section{REFERENCES}

1. Paladino VM, Miguel TS, Miguel BS, Pereira MM, Junior FA, Teixeira CG, Babinski CG, dos Anjos NK, da Costa DA, Martins DT, dos Santos BF. Mesenteric Adenitis as a Differential Appendicitis Diagnosis: Case Report. Journal of Biosciences and Medicines. 2018 Mar 6;6(3):26-30.

2. Toorenvliet B, Vellekoop A, Bakker R, Wiersma F, Mertens B, Merkus J, Breslau P, Hamming J. Clinical differentiation between acute appendicitis and acute mesenteric lymphadenitis in children. European Journal of Pediatric Surgery. 2011 Mar;21(02):120-3.

3. Mesenteric lymphadenitis, Journal of the American Medical Association. 1936.107(1): 41.
4. Wilensky AO, Hahn LJ. Mesenteric lymphadenitis. Ann Surg. 1926;83(6):812-826.

5. Ferguson G. Acute nonspecific mesenteric lymphadenitis: Possible Mechanism of Pain Illustrated by Two Cases. AMA archives of surgery. 1952 Dec 1;65(6):906-11.

6. Blattner RJ; Acute Mesenteric Lymphadenitis J Pediatr. 1969;74(3):479-81.

7. Helbling R, Conficconi E, Wyttenbach M, Benetti C, Simonetti GD, Bianchetti MG, Hamitaga F, Lava SA, Fossali EF, Milani GP. Acute nonspecific mesenteric lymphadenitis: more than "no need for surgery". BioMed research international. 2017 Feb $2 ; 2017$.

8. Helbling R, Conficconi E, Wyttenbach M, Benetti C, Simonetti GD, Bianchetti MG, Hamitaga F, Lava SA, Fossali EF, Milani GP. Acute nonspecific mesenteric lymphadenitis: more than "no need for surgery". BioMed research international. 2017 Feb $2 ; 2017$

9. Lee JH, Rhee PL, Lee JK, Lee KT, Son HJ, Kim JJ, Koh KC, Paik SW, Lee WJ, Lim HK, Rhee JC. The etiology and clinical characteristics of mesenteric adenitis in Korean adults. J Korean Med Sci. 1997 Apr 1;12(2):105-10.

10. Likitnukul S, Wongsawat J, Nunthapisud $P$. Appendicitis-like syndrome owing to mesenteric adenitis caused by Salmonella typhi. Annals of tropical paediatrics. 2002 Mar 1;22(1):97-9. 